\title{
Reconstruction of Digital Hologram by use of the Wavelet Transform
}

\author{
Jingang Zhong1,3 and Jiawen Weng2,1 \\ ${ }^{1}$ Department of Optoelectronic Engineering, Jinan University, \\ ${ }^{2}$ College of Science, South China Agricultural University, \\ ${ }^{3}$ Key Laboratory of Optoelectronic Information and Sensing Technologies of Guangdong \\ Higher Education Institutes, Jinan University,
}

China

\section{Introduction}

In combination with charge coupled device (CCD) sensor technology and image processing systems, real time dynamic analysis becomes one of the significant advantages of digital holography. In order to achieve the dynamic analysis, lots of holograms should be recorded and every hologram should be numerical reconstructed. The most popular numerical reconstruction methods for digital holography include the well-known Fresnel diffraction integral method, the angular spectrum method and the convolution-based method. In order to filter out the zero-order term, the twin image term and the parasitic interferences, the process of the spatial filtering must be carried out in these methods. When some noises and parasitic interferences are introduced into the hologram, the spectrum of the virtual image would be disturbed by some other spectrum. It brings difficulties to define the spatial filter because of the blurry boundary and non-regular distribution of the spectrum. Manual spatial filters are often employed. However, defining different manual spatial filters would consume plenty of time for dynamic analysis. Therefore, the process of the spatial filtering limits the application of the digital holography for the dynamic and automatic analysis.

The wavelet transform, a tool excelling for its multi-resolution and localization in the spacefrequency domains, is applied to the reconstruction of the object wave from the digital hologram in this chapter. Here the one-dimensional (1D) and two-dimensional (2D) Gabor wavelet transform (GWT) are employed for the analysis. By calculating the wavelet coefficients of the hologram at the ridge and the peak automatically, the object wave at the hologram plane can be reconstructed. At the same time the effect of the zero-order and the twin-image terms are eliminated without the process of the spatial filtering. The reconstruction approach for digital holography by use of the GWT is described in theory. And the experimental results are shown.

\section{Principle of the reconstruction technique employing the 1D-GWT}

\subsection{D-GWT}

The continuous one-dimensional wavelet transform is defined as follows:

$$
W(s, b)=\int_{-\infty}^{\infty} f(x) \psi_{s, b}^{*}(x) \mathrm{d} x=<f(x), \psi_{s, b}(x)>
$$




$$
\psi_{s, b}(x)=\frac{1}{s} \psi\left(\frac{x-b}{s}\right)
$$

where $s>0$ is the scale parameter related to the frequency; $b$ is the shift parameter related to the position; $f(x)$ is the signal to be analyzed; $\psi_{s, b}(x)$ is the analyzing wavelet obtained by shifting and scaling the mother wavelet $\psi(x) ;$ * indicates the complex conjugate.

The Gabor wavelet is employed as the mother wavelet. The Gabor wavelet function and its Fourier transform are given as:

$$
\left\{\begin{array}{l}
\psi(x)=\frac{1}{\sqrt[4]{\pi}} \sqrt{\frac{2 \pi}{\gamma}} \exp \left[-\frac{(2 \pi / \gamma)^{2} x^{2}}{2}+j 2 \pi x\right] \\
\psi(\omega)=\frac{\sqrt{2 \pi}}{\sqrt[4]{\pi}} \sqrt{\frac{\gamma}{2 \pi}} \exp \left[-\frac{(\gamma / 2 \pi)^{2}}{2}(\omega-2 \pi)^{2}\right]
\end{array}\right.
$$

where $\gamma=\pi \sqrt{2 / \ln 2}$. The Gabor wavelet may be considered as a Gaussian window centered at position $x=0$ and its Fourier transform centered at frequency $\omega=2 \pi$, as shown in Fig. 1. The function $\psi_{s, b}(x)$ is then centered around $x=b$ with a full width of half maximum $\Delta x=2 s$, and its Fourier transform is centered around $\omega=2 \pi / s$. The scale parameter $s$ controls the spatial and frequency resolution of the wavelet decomposition: A large $s$ corresponds to a stretched wave (high frequency resolution and low spatial resolution), whereas a small $s$ corresponds to a compressed wave (low frequency resolution and high spatial resolution). The wavelet coefficients $W(s, b)$ using the Gabor wavelet describes the distribution of the signal $f(x)$ on the space-frequency plane. Namely, it can be considered that each of the $W(s, b)$ contains the information about the local frequency $(\omega=2 \pi / s)$ component of the signal at the position $x=b$.

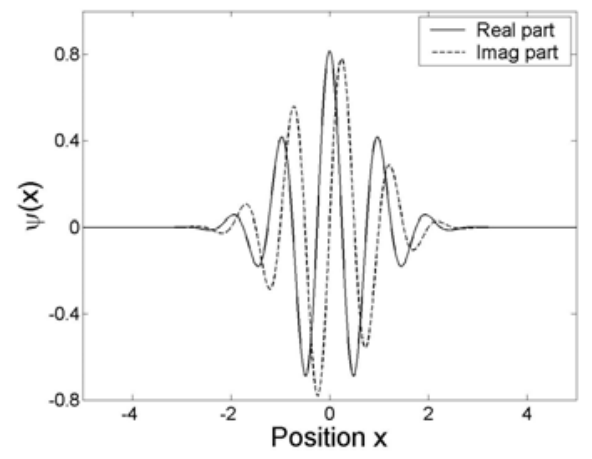

(a)

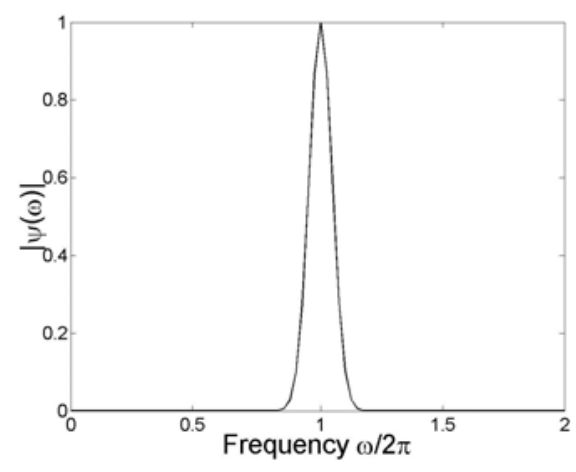

(b)

Fig. 1. (a) the Gabor wavelet and (b) its Fourier transform.

The reasons for the choice of the Gabor wavelet are as follows: (1) The Gabor wavelet consists of a sine wave and a cosine wave that have been modulated by a Gaussian function. (2) It can represent a complex sinusoidal function that correlates well with the sinusoidal 
characteristics of the interferogram. (3) The Gaussian function has the least spread in both domains of space and frequency.

Computing the 1D-GWT, the modulus of the wavelet coefficients can be obtained by:

$$
|W(s, b)|=\sqrt{\{\operatorname{imag}[W(s, b)]\}^{2}+\{\operatorname{real}[W(s, b)]\}^{2}}
$$

where $\operatorname{imag}[W(s, b)]$ and $\operatorname{real}[W(s, b)]$ indicate the imaginary part and the real part of the $W(s, b)$. At the position $b$, the ridge of the wavelet transform is defined as the location where the modulus $|W(s, b)|$ reaches its local maximum along the scaling direction $s$.

\subsection{Reconstruction principle by means of the 1D-GWT}

The hologram is created by the interference, in off-axis geometry, between two coherent waves: on one side the wave of interest, called object wave $O(x, y)$, coming from the object, and on the other side a reference wave $R(x, y)$ being plane:

$$
\begin{aligned}
& O(x, y)=o(x, y) \cdot \exp [j \phi(x, y)] \\
& R(x, y)=R_{0} \cdot \exp \left[j 2 \pi \frac{\sin \theta}{\lambda} x\right]
\end{aligned}
$$

where $(x, y)$ are the coordinates of the hologram plane; $o(x, y)$ and $R_{0}$ are the amplitude of the object and the reference waves; $\phi(x, y)$ is the phase of the object wave; $\lambda$ is the wavelength; $\theta$ is the angle between the propagation direction of the object and the reference waves. The intensity of the hologram $I(x, y)$ can be written as:

$$
\begin{aligned}
I(x, y)=R \cdot R^{*}+O \cdot O^{*}+O \cdot R^{*}+O^{*} \cdot R \\
=\left|R_{0}\right|^{2}+|o(x, y)|^{2}+R_{0} o(x, y) \exp \left\{j\left[-2 \pi \frac{\sin \theta}{\lambda} x+\phi(x, y)\right]\right\} \\
+R_{0} o(x, y) \exp \left\{-j\left[-2 \pi \frac{\sin \theta}{\lambda} x+\phi(x, y)\right]\right\}
\end{aligned}
$$

The first and the second terms form the zero-order term, the third and the fourth terms are the virtual and the real image terms respectively. Take one row of the hologram to analyze. The one-dimensional intensity distribution of the hologram can be written as:

$$
I(x)=A(x)+R_{0} o(x) \exp [j \varphi(x)]+R_{0} o(x) \exp [-j \varphi(x)]
$$

where $A(x)=\left|R_{0}\right|^{2}+|o(x)|^{2}$ and $\varphi(x)=-2 \pi \frac{\sin \theta}{\lambda} x+\phi(x)$. The phase $\varphi(x)$ can be expanded to a Taylor series around $b$ :

$$
\varphi(x)=\left[-2 \pi \frac{\sin \theta}{\lambda} b+\phi(b)\right]+\left[-2 \pi \frac{\sin \theta}{\lambda}+\phi^{\prime}(b)\right](x-b)+\frac{\phi^{\prime \prime}(b)}{2 !}(x-b)^{2}+\cdots
$$


When the higher order terms of $(x-b)$ are neglected, the phase $\varphi(x)$ can be expressed as:

$$
\varphi(x) \approx\left[-2 \pi \frac{\sin \theta}{\lambda} b+\phi(b)\right]+\left[-2 \pi \frac{\sin \theta}{\lambda}+\phi^{\prime}(b)\right](x-b)
$$

Setting $A(x) \approx A, o(x) \approx o$, the wavelet transform of $I(x)$ becomes:

$$
\begin{aligned}
W(s, b) & =\int_{-\infty}^{\infty} I(x) \psi_{s, b}^{*}(x) \mathrm{d} x \\
& =\int_{-\infty}^{\infty}\left\{A+R_{0} o \exp [j \varphi(x)]+R_{0} o \exp [-j \varphi(x)]\right\} \psi_{s, b}^{*}(x) \mathrm{d} x \\
& =W_{1}(s, b)+W_{2}(s, b)+W_{3}(s, b)
\end{aligned}
$$

Perform the above calculation by employing the Gabor wavelet as follows:

$$
\begin{aligned}
& W_{1}(s, b)=\int_{-\infty}^{\infty} A \cdot \psi_{s, b}^{*}(x) \mathrm{d} x \\
& =\frac{1}{s} \int_{-\infty}^{\infty} A \cdot \frac{1}{\sqrt[4]{\pi}} \sqrt{\frac{2 \pi}{\gamma}} \exp \left[-(2 \pi / \gamma)^{2} \frac{(x-b)^{2}}{2 s^{2}}\right] \exp \left(-j 2 \pi \frac{x-b}{s}\right) \mathrm{d} x \\
& \left(\operatorname{set} \frac{x-b}{s}=t\right) \\
& =A \cdot \frac{1}{\sqrt[4]{\pi}} \sqrt{\frac{2 \pi}{\gamma}} \int_{-\infty}^{\infty} \exp \left[-(2 \pi / \gamma)^{2} \frac{t^{2}}{2}\right] \exp (-j 2 \pi t) \mathrm{d} t \\
& =A \cdot \frac{1}{\sqrt[4]{\pi}} \sqrt{\frac{2 \pi}{\gamma}}\left\{\int_{-\infty}^{\infty} \exp \left[-(2 \pi / \gamma)^{2} \frac{t^{2}}{2}\right] \cos (2 \pi t) \mathrm{d} t-j \int_{-\infty}^{\infty} \exp \left[-(2 \pi / \gamma)^{2} \frac{t^{2}}{2}\right] \sin (2 \pi t) \mathrm{d} t\right\} \\
& =A \cdot \frac{1}{\sqrt[4]{\pi}} \sqrt{\frac{2 \pi}{\gamma}}\left\{2 \int_{0}^{\infty} \exp \left[-(2 \pi / \gamma)^{2} \frac{t^{2}}{2}\right] \cos (2 \pi t) \mathrm{d} t\right\} \\
& \left(\text { consider } \int_{0}^{\infty} \exp \left(-\xi^{2} x^{2}\right) \cos (\eta x) \mathrm{d} x=\frac{\exp \left[-\frac{\eta^{2}}{4 \xi^{2}}\right]}{2 \xi} \sqrt{\pi},(\xi>0)\right) \\
& =A \cdot \frac{1}{\sqrt[4]{\pi}} \sqrt{\frac{2 \pi}{\gamma}} 2 \frac{\exp \left[-\frac{(2 \pi)^{2}}{4 \frac{(2 \pi / \gamma)^{2}}{2}}\right]}{2 \sqrt{\frac{(2 \pi / \gamma)^{2}}{2}}} \sqrt{\pi} \\
& =\frac{\sqrt{\gamma}}{\sqrt[4]{\pi}} A \exp \left(-\frac{\gamma^{2}}{2}\right)
\end{aligned}
$$




$$
\begin{aligned}
W_{2}(s, b) & =\int_{-\infty}^{\infty} R_{0} o \exp [j \varphi(x)] \psi_{s, b}^{*}(x) \mathrm{d} x \\
& =\int_{-\infty}^{\infty} R_{0} o \exp \left\{j\left[-2 \pi \frac{\sin \theta}{\lambda} b+\phi(b)\right]\right\} \exp \left\{j\left[-2 \pi \frac{\sin \theta}{\lambda}+\phi^{\prime}(b)\right](x-b)\right\} \psi_{s, b}^{*}(x) \mathrm{d} x
\end{aligned}
$$

where,

$$
\begin{aligned}
& \int_{-\infty}^{\infty} \exp \left\{j\left[-2 \pi \frac{\sin \theta}{\lambda}+\phi^{\prime}(b)\right](x-b)\right\} \psi_{s, b}^{*}(x) \mathrm{d} x \\
& =\frac{1}{s} \int_{-\infty}^{\infty} \exp \left\{j\left[-2 \pi \frac{\sin \theta}{\lambda}+\phi^{\prime}(b)\right](x-b)\right\} \cdot \frac{1}{\sqrt[4]{\pi}} \sqrt{\frac{2 \pi}{\gamma}} \exp \left[-(2 \pi / \gamma)^{2} \frac{(x-b)^{2}}{2 s^{2}}\right] \exp \left(-j 2 \pi \frac{x-b}{s}\right) \mathrm{d} x \\
& \left(\operatorname{set} \frac{x-b}{s}=t\right) \\
& =\frac{1}{\sqrt[4]{\pi}} \sqrt{\frac{2 \pi}{\gamma}} \int_{-\infty}^{\infty} \exp \left\{j\left[-2 \pi \frac{\sin \theta}{\lambda}+\phi^{\prime}(b)\right] s t\right\} \cdot \exp \left[-(2 \pi / \gamma)^{2} \frac{t^{2}}{2}\right] \exp (-j 2 \pi t) \mathrm{d} t \\
& =\frac{1}{\sqrt[4]{\pi}} \sqrt{\frac{2 \pi}{\gamma}} \int_{-\infty}^{\infty} \exp \left[-(2 \pi / \gamma)^{2} \frac{t^{2}}{2}\right] \exp \left\{j 2 \pi t\left[\left(-\frac{\sin \theta}{\lambda}+\frac{\phi^{\prime}(b)}{2 \pi}\right) s-1\right]\right\} \mathrm{d} t \\
& =\frac{1}{\sqrt[4]{\pi}} \sqrt{\frac{2 \pi}{\gamma}} 2 \int_{0}^{\infty} \exp \left[-(2 \pi / \gamma)^{2} \frac{t^{2}}{2}\right] \cos \left\{2 \pi t\left[\left(-\frac{\sin \theta}{\lambda}+\frac{\phi^{\prime}(b)}{2 \pi}\right) s-1\right]\right\} \mathrm{d} t \\
& =\frac{1}{\sqrt[4]{\pi}} \sqrt{\frac{2 \pi}{\gamma}} 2 \frac{\exp \left\{-\frac{(2 \pi)^{2}\left[\left(-\frac{\sin \theta}{\lambda}+\frac{\phi^{\prime}(b)}{2 \pi}\right) s-1\right]^{2}}{4 \frac{(2 \pi / \gamma)^{2}}{2}}\right\}}{2 \sqrt{\frac{(2 \pi / \gamma)^{2}}{2}}} \sqrt{\pi} \\
& =\frac{\sqrt{\gamma}}{\sqrt[4]{\pi}} \exp \left\{-\frac{\gamma^{2}}{2}\left[\left(-\frac{\sin \theta}{\lambda}+\frac{\phi^{\prime}(b)}{2 \pi}\right) s-1\right]^{2}\right\}
\end{aligned}
$$

There is,

$$
\begin{aligned}
& W_{2}(s, b)=\frac{\sqrt{\gamma}}{\sqrt[4]{\pi}} R_{0} o \exp \left\{-\frac{\gamma^{2}}{2}\left[\left(-\frac{\sin \theta}{\lambda}+\frac{\phi^{\prime}(b)}{2 \pi}\right) s-1\right]^{2}\right\} \exp \left\{j\left[-2 \pi \frac{\sin \theta}{\lambda} b+\phi(b)\right]\right\} \\
& W_{3}(s, b)=\int_{-\infty}^{\infty} R_{0} o \exp [-j \varphi(x)] \psi_{s, b}^{*}(x) \mathrm{d} x \\
& =\int_{-\infty}^{\infty} R_{0} o \exp \left\{-j\left[-2 \pi \frac{\sin \theta}{\lambda} b+\phi(b)\right]\right\} \exp \left\{-j\left[-2 \pi \frac{\sin \theta}{\lambda}+\phi^{\prime}(b)\right](x-b)\right\} \psi_{s, b}^{*}(x) \mathrm{d} x
\end{aligned}
$$

where, 


$$
\int_{-\infty}^{\infty} \exp \left\{-j\left[-2 \pi \frac{\sin \theta}{\lambda}+\phi^{\prime}(b)\right](x-b)\right\} \psi_{s, b}^{*}(x) \mathrm{d} x=\frac{\sqrt{\gamma}}{\sqrt[4]{\pi}} \exp \left\{-\frac{\gamma^{2}}{2}\left[\left(-\frac{\sin \theta}{\lambda}+\frac{\phi^{\prime}(b)}{2 \pi}\right) s+1\right]^{2}\right\}
$$

There is,

$$
W_{3}(s, b)=\frac{\sqrt{\gamma}}{\sqrt[4]{\pi}} R_{0} o \exp \left\{-\frac{\gamma^{2}}{2}\left[\left(-\frac{\sin \theta}{\lambda}+\frac{\phi^{\prime}(b)}{2 \pi}\right) s+1\right]^{2}\right\} \exp \left\{-j\left[-2 \pi \frac{\sin \theta}{\lambda} b+\phi(b)\right]\right\}
$$

Therefore, it can be got as follows:

$$
\left\{\begin{array}{l}
W_{1}(s, b)=\frac{\sqrt{\gamma}}{\sqrt[4]{\pi}} A \exp \left(-\gamma^{2} / 2\right) \\
W_{2}(s, b)=\frac{\sqrt{\gamma}}{\sqrt[4]{\pi}} R_{0} o \exp \left\{-\frac{\gamma^{2}}{2}\left[\left(-\frac{\sin \theta}{\lambda}+\frac{\phi^{\prime}(b)}{2 \pi}\right) s-1\right]^{2}\right\} \exp \left\{j\left[-2 \pi \frac{\sin \theta}{\lambda} b+\phi(b)\right]\right\} \\
W_{3}(s, b)=\frac{\sqrt{\gamma}}{\sqrt[4]{\pi}} R_{0} o \exp \left\{-\frac{\gamma^{2}}{2}\left[\left(-\frac{\sin \theta}{\lambda}+\frac{\phi^{\prime}(b)}{2 \pi}\right) s+1\right]^{2}\right\} \exp \left\{-j\left[-2 \pi \frac{\sin \theta}{\lambda} b+\phi(b)\right]\right\}
\end{array}\right.
$$

Because of the feature of $s>0$, the modulus $|W(s, b)|$ reaches its maximum at:

$$
s=\frac{1}{-\frac{\sin \theta}{\lambda}+\frac{\phi^{\prime}(b)}{2 \pi}}
$$

Therefore, the wavelet coefficients at the ridge of the 1D-GWT, which is described as $W_{\text {ridge }}(b)$, become:

$$
\begin{aligned}
W_{\text {ridge }}(b)= & \frac{\sqrt{\gamma}}{\sqrt[4]{\pi}} A \exp \left(-\gamma^{2} / 2\right)+\frac{\sqrt{\gamma}}{\sqrt[4]{\pi}} R_{0} o \exp \left\{j\left[-2 \pi \frac{\sin \theta}{\lambda} b+\phi(b)\right]\right\} \\
& +\frac{\sqrt{\gamma}}{\sqrt[4]{\pi}} R_{0} o \exp \left(-2 \gamma^{2}\right) \exp \left\{-j\left[-2 \pi \frac{\sin \theta}{\lambda} b+\phi(b)\right]\right\}
\end{aligned}
$$

Here $\exp \left(-\gamma^{2} / 2\right)=\exp \left(-\pi^{2} / \ln 2\right) \approx 0$ and $\exp \left(-2 \gamma^{2}\right)=\exp \left(-4 \pi^{2} / \ln 2\right) \approx 0$. Note that the position parameter $b$ relates to $x$, so that Eqs. (21) can be rewritten as:

$$
W_{\text {ridge }}(x)=\frac{\sqrt{\gamma}}{\sqrt[4]{\pi}} R_{0} \operatorname{eexp}\left\{j\left[-2 \pi \frac{\sin \theta}{\lambda} x+\phi(x)\right]\right\}
$$

Multiplying the $W_{\text {ridge }}(x)$ by an ideal wave corresponding to a replica of the reference wave, the reconstructed wave $U_{\text {ridge }}(x)$ at the hologram plane is obtained as:

$$
U_{\text {ridge }}(x)=W_{\text {ridge }}(x) R_{0} \exp \left[j 2 \pi \frac{\sin \theta}{\lambda} x\right]=\frac{\sqrt{\gamma}}{\sqrt[4]{\pi}} R_{0}^{2} o \exp [j \phi(x)]
$$


A conclusion can be obtained from Eqs. (23) that the reconstructed wave at the ridge of the 1D-GWT is equal to the object wave at the hologram plane multiplied by a constant coefficient. Therefore the numerical reconstruction of the object wave can be carried out by means of the 1D-GWT. At the same time the effect of the zero-order and the twin-image terms are eliminated without the process of the spatial filtering.

It should be noted that the conclusion here is obtained by assuming the higher order terms of $(x-b)$ in Eqs. (9) being neglected. In the following two cases this approximation can be satisfied. First, if $\phi^{\prime}(x)$ is of slow variation, it can be set that $\phi^{\prime \prime}(x) \approx \phi^{\prime \prime \prime}(x) \approx \cdots \approx 0$, subsequently the higher order terms of $(x-b)$ can be neglected. The phase of the object wave $\phi(x)$ corresponds to the optical path length (OPL) when a plane wave propagates through a phase object. Therefore, the slow variation of $\phi^{\prime}(x)$ denotes that the first derivative of OPL is of slow variation, or the first derivative of refraction-index distribution of the phase object is of slow variation in physics. Second, because the function $\psi_{s, b}(x)$ is concentrated around $x=b$ with a full width of half maximum $\Delta x=2 s$, the higher order terms of $(x-b)$ can be neglected when $(x-b)<1$ in the full width.

The reconstructed wave at the object plane perpendicular to the propagation axis can be calculated by the Fresnel diffraction integral operation or by the angular spectrum method. Suppose that the wave front at the hologram plane is described as $E(x, y ; 0)$, and its angular spectrum $A(\xi, \eta ; 0)=\mathfrak{I}\{E(x, y ; 0)\}$ can be obtained by taking the Fourier transform. Here $\mathfrak{J}\{\}$ denotes the Fourier transform, and $(\xi, \eta)$ are the corresponding spatial frequencies of $(x, y)$. The angular spectrum of the reconstructed wave at the plane $z$ perpendicular to the propagation axis, $A(\xi, \eta ; z)$, can be calculated from $A(\xi, \eta ; 0)$ as:

$$
A(\xi, \eta ; z)=A(\xi, \eta ; 0) \cdot \exp \left[j \frac{2 \pi z}{\lambda} \sqrt{1-(\lambda \xi)^{2}-(\lambda \eta)^{2}}\right]
$$

Subsequently the reconstructed wave can be obtained by taking the inverse Fourier transform as $E^{\prime}\left(x^{\prime}, y^{\prime} ; z\right)=\mathfrak{I}^{-1}\{A(\xi, \eta ; z)\}$, where $\mathfrak{I}^{-1}\{\}$ denotes the inverse Fourier transform.

\section{Principle of the reconstruction technique employing the 2D-GWT}

\subsection{D-GWT}

There is a deficiency of the 1D-GWT method that the two-dimensional hologram must be divided into a series of one-dimensional signals along $x$ direction when computing the 1DGWT on $x$ direction with $y$ fixed. Thereby, the spatial frequency on $y$ direction would be lost. It means that it would fail to analyze the fringes along $x$ direction. Especially when there are some interference fringes of the hologram are along $x$ direction but others along $y$ direction proposed, or there are some parasitic interference signals along $x$ direction, the two-dimensional wavelet transform is necessary.

The continuous two-dimensional wavelet transform is defined as follows:

$$
\begin{gathered}
W_{f}(s, \theta, a, b)=\iint_{-\infty}^{\infty} f(x, y) \psi_{s, \theta}^{*}(x, y, a, b) \mathrm{d} x \mathrm{~d} y \\
\psi_{s, \theta}(x, y, a, b)=\frac{1}{s^{2}} \psi\left(\frac{x-a}{s}, \frac{x-b}{s}, \theta\right)
\end{gathered}
$$


where $s(>0)$ and $\theta$ are the scale and the rotation parameters related to the frequency; $(a, b)$ is the shift parameter related to the position; $f(x, y)$ is a two-dimensional real signal to be analyzed; $\psi_{s, \theta}(x, y, a, b)$ is the analyzing wavelet obtained by shifting, scaling and rotating the mother wavelet $\psi(x, y)$, and it can be considered as a spatial window; * indicates the complex conjugate. Here the one-dimensional Gabor wavelet is extended to the twodimensional situation to create a two-dimensional Gabor wavelet as a mother wavelet for its optimal joint space and frequency resolution for a signal. The two-dimensional Gabor wavelet function is given as:

$$
\psi(x, y)=\frac{1}{\sqrt[4]{\pi}} \sqrt{\frac{2 \pi}{\gamma}} \exp \left[-\frac{(2 \pi / \gamma)^{2}\left(x^{2}+y^{2}\right)}{2}+j 2 \pi(x+y)\right]
$$

where $\gamma=\pi \sqrt{2 / \ln 2}$, and $j$ is the imaginary unit. A series of analyzing wavelets, namely "daughter wavelets", are built up by shifting, scaling and rotating the two-dimensional Gabor wavelet, namely "mother wavelet", as follows:

$$
\begin{gathered}
\psi\left(\frac{x-a}{s}, \frac{x-b}{s}, \theta\right)=\frac{1}{\sqrt[4]{\pi}} \sqrt{\frac{2 \pi}{\gamma}} \exp \left\{-\frac{(2 \pi / \gamma)^{2}\left[(x-a)^{2}+(y-b)^{2}\right]}{2 s^{2}}\right\} \\
\cdot \exp \left\{j 2 \pi \frac{(x-a) \cos \theta+(y-b) \sin \theta}{s}\right\}
\end{gathered}
$$

Computing the 2D-GWT of the signal $f(x, y)$, the modulus $\left|W_{f}(s, \theta, a, b)\right|$ and the phase $\psi(s, \theta, a, b)$ of the wavelet coefficients $W_{f}(s, \theta, a, b)$ are obtained as:

$$
\begin{gathered}
\left|W_{f}(s, \theta, a, b)\right|=\sqrt{\left\{\operatorname{imag}\left[W_{f}(s, \theta, a, b)\right]\right\}^{2}+\left\{\operatorname{real}\left[W_{f}(s, \theta, a, b)\right]\right\}^{2}} \\
\psi(s, \theta, a, b)=\arctan \left\{\frac{\operatorname{imag}\left[W_{f}(s, \theta, a, b)\right]}{\operatorname{real}\left[W_{f}(s, \theta, a, b)\right]}\right\}
\end{gathered}
$$

where $\operatorname{imag}\left[W_{f}(s, \theta, a, b)\right]$ and real $\left[W_{f}(s, \theta, a, b)\right]$ indicate the imaginary part and the real part of the $W_{f}(s, \theta, a, b)$. The result of the 2D-GWT is a four-dimensional array related to a parameter $a$, a parameter $b$, a scale parameter $s$ and a rotation angle parameter $\theta$. Fig. 2 shows an illustration of a four-dimensional map of size $512 \times 512$ pixel, in schematic form, for a specific example of a 2D-GWT at five different scale values $\{2,3,4,5,6\}$ and three different rotation angle values $\left\{0^{\circ}, 30^{\circ}, 60^{0}\right\}$.

At a position $(a, b)$, the peak of the 2D-GWT is defined as the location where the modulus $\left|W_{f}(s, \theta, a, b)\right|$ reaches its local maximum along both the scaling direction $s$ and the rotating direction $\theta$. The determination of the peak of the 2D-GWT can be carried out by the following process. First, an array is constructed by picking up the modulus of the 2D-GWT 


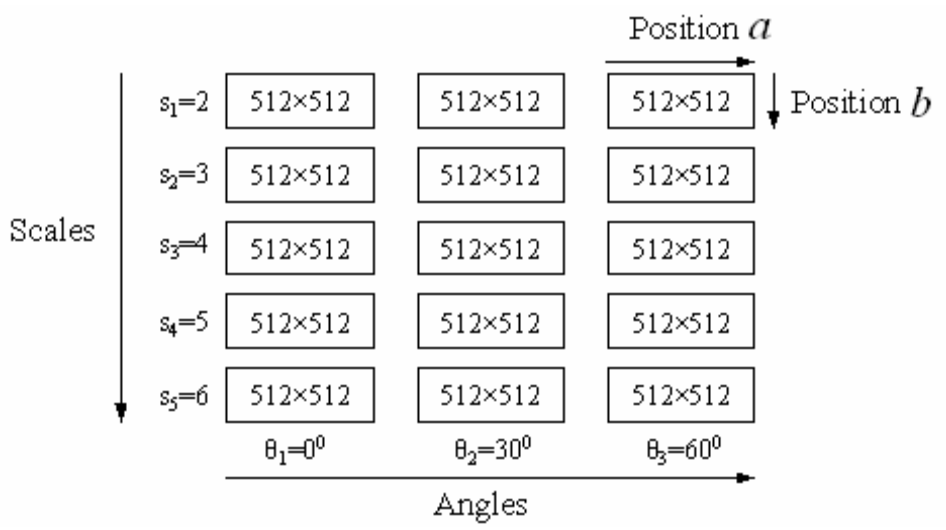

Fig. 2. Illustration of a four-dimensional array produced by the 2D-GWT.

coefficients corresponding to the position $(a, b)$ at each scale and rotation angle value. For the above specific example including five different scale values and three different rotation angle values, a array of size $5 \times 3$ is obtained as shown in Fig. 3. Second, the maximum value of this array is found which is pointed out by an arrow in Fig. 3. Finally, the corresponding scale value and rotation angle value are determined, and the peak of the 2D-GWT at the position $(a, b)$ is determined.

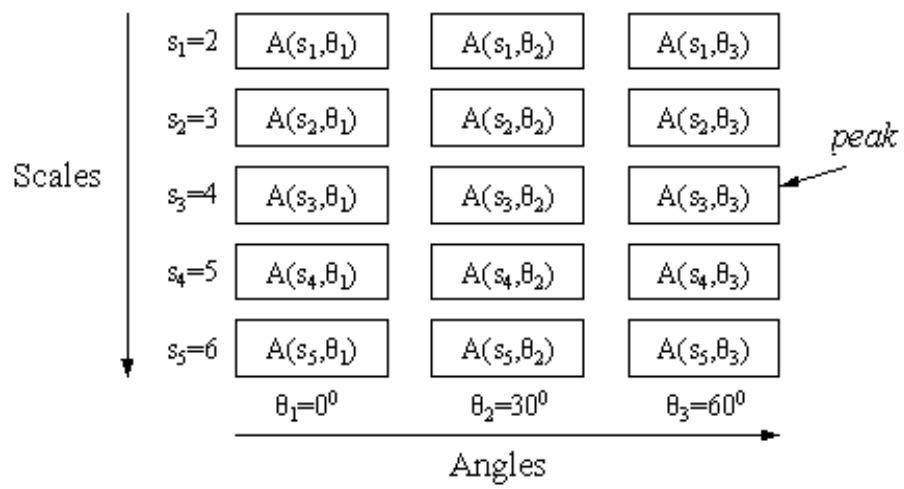

Fig. 3. Illustration of the peak of the 2D-GWT.

\subsection{Reconstruction principle by means of the 2D-GWT}

The object wave $O(x, y)$ and the reference wave $R(x, y)$ being plane can be written as:

$$
\begin{gathered}
O(x, y)=o(x, y) \cdot \exp [j \phi(x, y)] \\
R(x, y)=R_{0} \cdot \exp \left[j \frac{2 \pi}{\lambda}(x \cos \alpha+y \cos \beta)\right]
\end{gathered}
$$


$\alpha$ and $\beta$ are the angles between the propagation direction of the object wave and the reference wave on $x$ and $y$ direction respectively. The intensity of the hologram $I(x, y)$ can be written as:

$$
\begin{aligned}
I(x, y)=R \cdot R^{*}+O \cdot O^{*} & +O \cdot R^{*}+O^{*} \cdot R \\
=\left|R_{0}\right|^{2}+|o(x, y)|^{2}+R_{0} o(x, y) \exp \left\{j\left[-\frac{2 \pi}{\lambda}(x \cos \alpha+y \cos \beta)+\phi(x, y)\right]\right\} & \\
& +R_{0} o(x, y) \exp \left\{-j\left[-\frac{2 \pi}{\lambda}(x \cos \alpha+y \cos \beta)+\phi(x, y)\right]\right\}
\end{aligned}
$$

The first and the second terms form the zero-order term, namely the background intensity, which is written as $A(x, y)=\left|R_{0}\right|^{2}+|o(x, y)|^{2}$. The third and the fourth terms are the virtual and the real image terms respectively. Defined the phase of the hologram as follows:

$$
\varphi(x, y)=-\frac{2 \pi}{\lambda}(x \cos \alpha+y \cos \beta)+\phi(x, y)
$$

The intensity distribution of the hologram can be written as:

$$
I(x, y)=A(x, y)+R_{0} o(x, y) \exp [j \varphi(x, y)]+R_{0} o(x, y) \exp [-j \varphi(x, y)]
$$

The phase $\varphi(x, y)$ can be expanded to a two-dimensional Taylor series around $(a, b)$ :

$$
\begin{aligned}
\varphi(x, y)=\varphi(a, b) & +\left[\left.(x-a) \frac{\partial \varphi(x, y)}{\partial x}\right|_{x=a, y=b}+\left.(y-b) \frac{\partial \varphi(x, y)}{\partial y}\right|_{x=a, y=b}\right] \\
& +\frac{1}{2 !}\left[\left.(x-a)^{2} \frac{\partial^{2} \varphi(x, y)}{\partial x^{2}}\right|_{x=a, y=b}+\left.(y-b)^{2} \frac{\partial^{2} \varphi(x, y)}{\partial x^{2}}\right|_{x=a, y=b}\right]+\ldots
\end{aligned}
$$

where $\frac{\partial}{\partial x}$ and $\frac{\partial}{\partial y}$ represent the partial differential operation. Assuming $\frac{\partial[\varphi(x, y]}{\partial x}$ and $\frac{\partial[\varphi(x, y]}{\partial y}$ are of slow variation, so that the higher order terms of the Taylor series can be neglected. The phase $\varphi(x, y)$ can be expressed as:

$$
\varphi(x, y)=\varphi(a, b)+\left[\left.(x-a) \frac{\partial \varphi(x, y)}{\partial x}\right|_{x=a, y=b}+\left.(y-b) \frac{\partial \varphi(x, y)}{\partial y}\right|_{x=a, y=b}\right]
$$


The instantaneous frequency is defined as follows:

$$
\left\{\begin{array}{l}
f_{x}=\frac{1}{2 \pi} \frac{\partial[\varphi(x, y)]}{\partial x} \\
f_{y}=\frac{1}{2 \pi} \frac{\partial[\varphi(x, y)]}{\partial y}
\end{array}\right.
$$

And the spatial frequency is:

$$
\left\{\begin{array}{l}
f_{x}=\frac{\cos \alpha}{T} \\
f_{y}=\frac{\cos \beta}{T}=\frac{\sin \alpha}{T}
\end{array}\right.
$$

where $T$ represents the spatial period of the hologram pattern around the position $(a, b)$. Therefore, Eqs. (37) can be rewritten as:

$$
\varphi(x, y)=\varphi(a, b)+2 \pi \frac{(x-a) \cos \alpha+(y-b) \sin \alpha}{T}
$$

And the intensity of the hologram expressed by Eqs. (35) can be rewritten as:

$$
\begin{aligned}
I(x, y)=A(x, y) & +R_{0} o(x, y) \exp \left\{j\left[2 \pi \frac{(x-a) \cos \alpha+(y-b) \sin \alpha}{T}+\varphi(a, b)\right]\right\} \\
& +R_{0} o(x, y) \exp \left\{-j\left[2 \pi \frac{(x-a) \cos \alpha+(y-b) \sin \alpha}{T}+\varphi(a, b)\right]\right\}
\end{aligned}
$$

Because of the reference wave being plane, $R_{0}$ is constant. And the phase object can be considered to be nearly transparent, i.e. it does not modulate the intensity of the illuminated wave but the phase, therefore it can be set that $o(x, y) \approx 0$ and $A(x, y) \approx A$. For the nonintensity-only object, when the amplitude of the object wave $o(x, y)$ is of slow variation compared with the phase term $\exp [j \phi(x, y)]$, we can set a slowly-varying-amplitude approximation $o(x, y) \approx 0$. This approximation is widely adopted in nonlinear optics. And it can be satisfied for the non-intensity-only objects easily, because when the optical path difference $\delta p$ changes one wavelength, i.e. $\delta p=\lambda$, the phase would changes $(2 \pi / \lambda) \delta p=2 \pi$ and $\exp [j \phi(x, y)]$ would has one periodic variation. For the intensity-only object, it seems that this approximation cannot be satisfied. However, there is not strict intensity-only wave due to the diffraction effect. Therefore the mentioned approximation condition can be satisfied in general. Setting $o(x, y) \approx 0, A(x, y) \approx A$, and computing the 2DGWT of $I(x, y)$ becomes: 


$$
\begin{aligned}
W(s, \theta, a, b) & =\iint_{-\infty}^{\infty} I(x, y) \psi_{s, \theta}^{*}(x, y, a, b) \mathrm{d} x \mathrm{~d} y \\
& =\iint_{-\infty}^{\infty}\left\{A+R_{0} o \exp [j \varphi(x, y)]+R_{0} o \exp [-j \varphi(x, y)]\right\} \psi_{s, \theta}^{*}(x, y, a, b) \mathrm{d} x \mathrm{~d} y \\
& =W_{1}(s, \theta, a, b)+W_{2}(s, \theta, a, b)+W_{3}(s, \theta, a, b)
\end{aligned}
$$

Performing the above calculation by employing the two-dimensional Gabor wavelet as follows:

$$
\begin{aligned}
& W_{1}(s, \theta, a, b)=\iint_{-\infty}^{\infty} A \cdot \psi_{s, \theta}^{*}(x, y, a, b) \mathrm{d} x \mathrm{~d} y \\
& =\frac{1}{s^{2}} \iint_{-\infty}^{\infty} A \cdot \frac{1}{\sqrt[4]{\pi}} \sqrt{\frac{2 \pi}{\gamma}} \exp \left\{-\frac{(2 \pi / \gamma)^{2}\left[(x-a)^{2}+(y-b)^{2}\right]}{2 s^{2}}\right\} \cdot \exp \left\{-j 2 \pi \frac{(x-a) \cos \theta+(y-b) \sin \theta}{s}\right\} \mathrm{d} x \mathrm{~d} y \\
& =A \cdot \frac{1}{\sqrt[4]{\pi}} \sqrt{\frac{2 \pi}{\gamma}} \int_{-\infty}^{\infty} \frac{1}{s} \exp \left[-\frac{(2 \pi / \gamma)^{2}(x-a)^{2}}{2 s^{2}}\right] \exp \left[-j 2 \pi \frac{(x-a)}{s} \cos \theta\right] \mathrm{d} x \\
& \cdot \int_{-\infty}^{\infty} \frac{1}{s} \exp \left[-\frac{(2 \pi / \gamma)^{2}(y-b)^{2}}{2 s^{2}}\right] \exp \left[-j 2 \pi \frac{(y-b)}{s} \sin \theta\right] \mathrm{d} y \\
& \left(\operatorname{set} \frac{x-a}{s}=u, \frac{y-b}{s}=v\right) \\
& =A \cdot \frac{1}{\sqrt[4]{\pi}} \sqrt{\frac{2 \pi}{\gamma}} \int_{-\infty}^{\infty} \exp \left[-\frac{(2 \pi / \gamma)^{2} u^{2}}{2}\right] \exp [-j 2 \pi u \cos \theta] \mathrm{d} u \cdot \int_{-\infty}^{\infty} \exp \left[-\frac{(2 \pi / \gamma)^{2} v^{2}}{2}\right] \exp [-j 2 \pi v \sin \theta] \mathrm{d} v \\
& =A \cdot \frac{1}{\sqrt[4]{\pi}} \sqrt{\frac{2 \pi}{\gamma}}\left\{2 \int_{0}^{\infty} \exp \left[-\frac{(2 \pi / \gamma)^{2} u^{2}}{2}\right] \cos [2 \pi u \cos \theta] \mathrm{d} u\right\} \cdot\left\{2 \int_{0}^{\infty} \exp \left[-\frac{(2 \pi / \gamma)^{2} v^{2}}{2}\right] \cos [2 \pi v \sin \theta] \mathrm{d} v\right\} \\
& =A \cdot \frac{1}{\sqrt[4]{\pi}} \sqrt{\frac{2 \pi}{\gamma}}\left\{2 \frac{\exp \left[-\frac{(2 \pi \cos \theta)^{2}}{4 \frac{(2 \pi / \gamma)^{2}}{2}}\right]}{2 \sqrt{\frac{(2 \pi / \gamma)^{2}}{2}}} \sqrt{\pi}\right\} \cdot\left\{2 \frac{\exp \left[-\frac{(2 \pi \sin \theta)^{2}}{4 \frac{(2 \pi / \gamma)^{2}}{2}}\right]}{2 \sqrt{\frac{(2 \pi / \gamma)^{2}}{2}}} \sqrt{\pi}\right\} \\
& =\frac{\sqrt{\gamma^{3}}}{\sqrt[4]{4 \pi^{3}}} A \exp \left(-\frac{\gamma^{2}}{2}\right) \\
& W_{2}(s, \theta, a, b)=\iint_{-\infty}^{\infty} R_{0} o \exp [j \varphi(x)] \cdot \psi_{s, \theta}^{*}(x, y, a, b) \mathrm{d} x \mathrm{~d} y \\
& =\iint_{-\infty}^{\infty} R_{0} o \exp \left[j 2 \pi \frac{(x-a) \cos \alpha+(y-b) \sin \alpha}{T}\right] \exp [j \varphi(a, b)] \cdot \psi_{s, \theta}^{*}(x, y, a, b) \mathrm{d} x \mathrm{~d} y
\end{aligned}
$$


where

$$
\begin{aligned}
& \iint_{-\infty}^{\infty} \exp \left[j 2 \pi \frac{(x-a) \cos \alpha+(y-b) \sin \alpha}{T}\right] \cdot \psi_{s, \theta}^{*}(x, y, a, b) \mathrm{d} x \mathrm{~d} y \\
& =\frac{1}{s^{2}} \iint_{-\infty}^{\infty} \exp \left[j 2 \pi \frac{(x-a) \cos \alpha+(y-b) \sin \alpha}{T}\right] \\
& \cdot \frac{1}{\sqrt[4]{\pi}} \sqrt{\frac{2 \pi}{\gamma}} \exp \left\{-\frac{(2 \pi / \gamma)^{2}\left[(x-a)^{2}+(y-b)^{2}\right]}{2 s^{2}}\right\} \exp \left\{-j 2 \pi \frac{(x-a) \cos \theta+(y-b) \sin \theta}{s}\right\} \mathrm{d} x \mathrm{~d} y \\
& =\frac{1}{\sqrt[4]{\pi}} \sqrt{\frac{2 \pi}{\gamma}} \int_{-\infty}^{\infty} \frac{1}{s} \exp \left[j 2 \pi \frac{(x-a) \cos \alpha}{T}\right] \exp \left[-\frac{(2 \pi / \gamma)^{2}(x-a)^{2}}{2 s^{2}}\right] \exp \left[-j 2 \pi \frac{(x-a)}{s} \cos \theta\right] \mathrm{d} x \\
& \cdot \int_{-\infty}^{\infty} \frac{1}{s} \exp \left[j 2 \pi \frac{(y-b) \sin \alpha}{T}\right] \exp \left[-\frac{(2 \pi / \gamma)^{2}(y-b)^{2}}{2 s^{2}}\right] \exp \left[-j 2 \pi \frac{(y-b)}{s} \sin \theta\right] \mathrm{d} y \\
& \left(\operatorname{set} \frac{x-a}{s}=u, \frac{y-b}{s}=v\right) \\
& =\frac{1}{\sqrt[4]{\pi}} \sqrt{\frac{2 \pi}{\gamma}} \int_{-\infty}^{\infty} \exp \left[-\frac{(2 \pi / \gamma)^{2} u^{2}}{2}\right] \exp \left[j 2 \pi \frac{s}{T} u \cos \alpha\right] \exp [-j 2 \pi u \cos \theta] \mathrm{d} u \\
& \cdot \int_{-\infty}^{\infty} \exp \left[-\frac{(2 \pi / \gamma)^{2} v^{2}}{2}\right] \exp \left[j 2 \pi \frac{s}{T} v \sin \alpha\right] \exp [-j 2 \pi v \sin \theta] \mathrm{d} v \\
& =\frac{1}{\sqrt[4]{\pi}} \sqrt{\frac{2 \pi}{\gamma}}\left\{2 \int_{0}^{\infty} \exp \left[-\frac{(2 \pi / \gamma)^{2} u^{2}}{2}\right] \cos \left[2 \pi\left(\frac{s}{T} \cos \alpha-\cos \theta\right) u\right] \mathrm{d} u\right\} \\
& \left\{2 \int_{0}^{\infty} \exp \left[-\frac{(2 \pi / \gamma)^{2} v^{2}}{2}\right] \cos \left[2 \pi\left(\frac{s}{T} \sin \alpha-\sin \theta\right) v\right] \mathrm{d} v\right\} \\
& =\frac{1}{\sqrt[4]{\pi}} \sqrt{\frac{2 \pi}{\gamma}}\left\{\frac{\exp \left[-\frac{4 \pi^{2}\left(\frac{s}{T} \cos \alpha-\cos \theta\right)^{2}}{4 \frac{(2 \pi / \gamma)^{2}}{2}}\right]}{2 \sqrt{\frac{(2 \pi / \gamma)^{2}}{2}}} \sqrt{\pi}\right\} \cdot\left\{\frac{\exp \left[-\frac{4 \pi^{2}\left(\frac{s}{T} \sin \alpha-\sin \theta\right)^{2}}{4 \frac{(2 \pi / \gamma)^{2}}{2}}\right]}{2 \sqrt{\frac{(2 \pi / \gamma)^{2}}{2}}} \sqrt{\pi}\right\} \\
& =\frac{\sqrt{\gamma^{3}}}{\sqrt[4]{4 \pi^{3}}} \exp \left\{-\frac{\gamma^{2}}{2}\left[\left(\frac{s}{T}-1\right)^{2}+2 \frac{s}{T}(1-\cos (\alpha-\theta))\right]\right\}
\end{aligned}
$$

There is, 


$$
W_{2}(s, \theta, a, b)=\frac{\sqrt{\gamma^{3}}}{\sqrt[4]{4 \pi^{3}}} R_{0} o \exp \left\{-\frac{\gamma^{2}}{2}\left[\left(\frac{s}{T}-1\right)^{2}+2 \frac{s}{T}(1-\cos (\alpha-\theta))\right]\right\} \exp [j \varphi(a, b)]
$$

$$
\begin{aligned}
W_{3}(s, \theta, a, b) & =\iint_{-\infty}^{\infty} R_{0} o \exp [-j \varphi(x)] \cdot \psi_{s, \theta}^{*}(x, y, a, b) \mathrm{d} x \mathrm{~d} y \\
& =\iint_{-\infty}^{\infty} R_{0} o \exp \left[-j 2 \pi \frac{(x-a) \cos \alpha+(y-b) \sin \alpha}{T}\right] \exp [-j \varphi(a, b)] \cdot \psi_{s, \theta}^{*}(x, y, a, b) \mathrm{d} x \mathrm{~d} y
\end{aligned}
$$

where

$$
\begin{aligned}
& \iint_{-\infty}^{\infty} \exp \left[-j 2 \pi \frac{(x-a) \cos \alpha+(y-b) \sin \alpha}{T}\right] \cdot \psi_{s, \theta}^{*}(x, y, a, b) \mathrm{d} x \mathrm{~d} y \\
= & \frac{\sqrt{\gamma^{3}}}{\sqrt[4]{4 \pi^{3}}} \exp \left\{-\frac{\gamma^{2}}{2}\left[\left(\frac{s}{T}+1\right)^{2}-2 \frac{s}{T}(1-\cos (\alpha-\theta))\right]\right\}
\end{aligned}
$$

There is,

$$
W_{3}(s, \theta, a, b)=\frac{\sqrt{\gamma^{3}}}{\sqrt[4]{4 \pi^{3}}} R_{0} o \exp \left\{-\frac{\gamma^{2}}{2}\left[\left(\frac{s}{T}+1\right)^{2}-2 \frac{s}{T}(1-\cos (\alpha-\theta))\right]\right\} \exp [-j \varphi(a, b)]
$$

Therefore, it can be got as follows:

$$
\left\{\begin{array}{l}
W_{1}(s, \theta, a, b)=\frac{\sqrt{\gamma^{3}}}{\sqrt[4]{4 \pi^{3}}} A \exp \left(-\frac{\gamma^{2}}{2}\right) \\
W_{2}(s, \theta, a, b)=\frac{\sqrt{\gamma^{3}}}{\sqrt[4]{4 \pi^{3}}} R_{0} o \exp \left\{-\frac{\gamma^{2}}{2}\left[\left(\frac{s}{T}-1\right)^{2}+2 \frac{s}{T}(1-\cos (\alpha-\theta))\right]\right\} \exp \{j \varphi(a, b)\} \\
W_{3}(s, \theta, a, b)=\frac{\sqrt{\gamma^{3}}}{\sqrt[4]{4 \pi^{3}}} R_{0} o \exp \left\{-\frac{\gamma^{2}}{2}\left[\left(\frac{s}{T}+1\right)^{2}-2 \frac{s}{T}(1-\cos (\alpha-\theta))\right]\right\} \exp \{-j \varphi(a, b)\}
\end{array}\right.
$$

Because of the feature of $s>0$, the modulus $\left|W_{f}(s, \theta, a, b)\right|$ reaches its maximum at $s=T$ and $\theta=\alpha$ in theory according to Eqs. (50). It means that not only the spatial frequency on $x$ direction but also the spatial frequency on $y$ direction, which cannot be obtained by the 1DGWT, are determined according to Eqs. (39). Therefore, the wavelet coefficients at the peak of the 2D-GWT are obtained according to not only $f_{x}$ but also $f_{y}$, which is described as $W_{\text {peak }}(a, b)$ :

$$
\begin{gathered}
W_{\text {peak }}(a, b)=\frac{\sqrt{\gamma^{3}}}{\sqrt[4]{4 \pi^{3}}} A \exp \left(-\gamma^{2} / 2\right)+\frac{\sqrt{\gamma^{3}}}{\sqrt[4]{4 \pi^{3}}} R_{0} o \exp \{j \varphi(a, b)\} \\
+\frac{\sqrt{\gamma^{3}}}{\sqrt[4]{4 \pi^{3}}} R_{0} o \exp \left(-2 \gamma^{2}\right) \exp \{-j \varphi(a, b)\}
\end{gathered}
$$


Here $\exp \left(-\gamma^{2} / 2\right)=\exp \left(-\pi^{2} / \ln 2\right) \approx 0$ and $\exp \left(-2 \gamma^{2}\right)=\exp \left(-4 \pi^{2} / \ln 2\right) \approx 0$. Note that the position parameter $(a, b)$ relates to $(x, y)$, so that Eqs. (51) can be rewritten as:

$$
W_{\text {peak }}(x, y)=\frac{\sqrt{\gamma^{3}}}{\sqrt[4]{4 \pi^{3}}} R_{0} o \exp \left\{j\left[-\frac{2 \pi}{\lambda}(x \cos \alpha+y \cos \beta)+\phi(x, y)\right]\right\}
$$

Multiplying the $W_{\text {peak }}(x, y)$ by an ideal wave corresponding to a replica of the reference wave, the reconstructed wave $U_{\text {peak }}(x, y)$ at the hologram plane is obtained as:

$$
\begin{aligned}
U_{\text {peak }}(x, y) & =W_{\text {peak }}(x, y) R_{0} \exp \left[j \frac{2 \pi}{\lambda}(x \cos \alpha+y \cos \beta)\right] \\
& =\frac{\sqrt{\gamma^{3}}}{\sqrt[4]{4 \pi^{3}}} R_{0}^{2} o \exp [\phi(x, y)]
\end{aligned}
$$

A conclusion can be obtained from Eqs. (53) that the reconstructed wave at the peak of the 2D-GWT is equal to the object wave at the hologram plane multiplied by a constant coefficient. Therefore the numerical reconstruction of the object wave, including not only the amplitude but also the phase information, can be carried out by means of the 2D-GWT. At the same time the effect of the zero-order and the twin-image terms is eliminated without the process of the spatial filtering. The reconstructed wave at the object plane perpendicular to the propagation axis can be calculated by the Fresnel diffraction integral operation or by the angular spectrum method, which is described by Eqs. (24).

\section{Experimental result}

The system depicted in Fig. 4 is analogous to a Mach-Zehnder interferometer. A He-Ne laser beam, followed by a beam enlarger (BE), is separated into two beams by a beam splitter BS1. One beam serves as a reference wave, and another beam illuminates the biological specimen. A microscope objective MO1 collects the object wave transmitted by the specimen and produces a magnified image of the specimen at a distance behind the CCD camera.

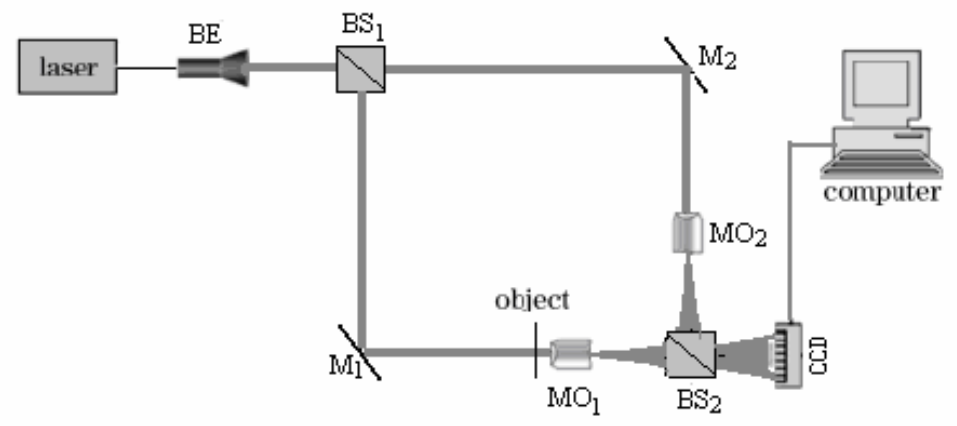

Fig. 4. Apparatus for digital holography. (BE: beam enlarger; BS1, BS2: beam splitter; M1, M2: mirror; MO1, MO2: microscope objective; CCD: charge coupled device.) 
Another MO2 is placed in the reference arm to get the matching wave front curvatures. The beam splitter BS2 placed in front of the CCD camera combines the two beams, and the holograms are recorded by the CCD camera. The slight angle is introduced between the object and the reference beams by tilting the beam splitter BS2 for the off-axis holography.

An onion specimen placed between the slide and the cover slide is illuminated by the laser beam. Fig. 5(a) shows the image of the onion specimen recorded by the CCD camera when the reference wave is turn off. Fig. $5(\mathrm{~b})$ is the hologram with the onion specimen recorded by the CCD camera of $512 \times 512$ pixels. In order to compensate for phase aberrations and image distortion, a reference hologram without the presence of any specimen is also recorded, as shown in Fig. 5(c). Fig. 5(d) is the spectrum of the hologram with the specimen on the logarithmic coordinates.

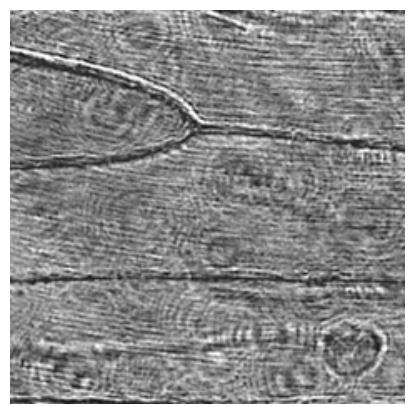

(a)

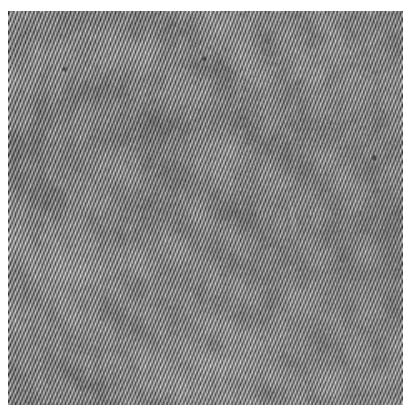

(c)

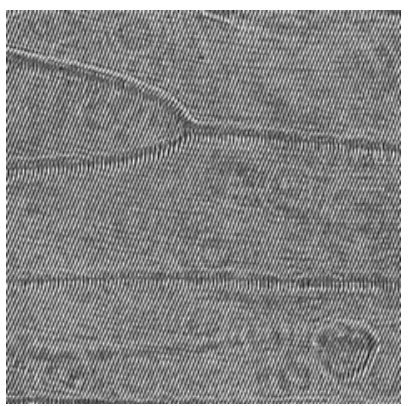

(b)

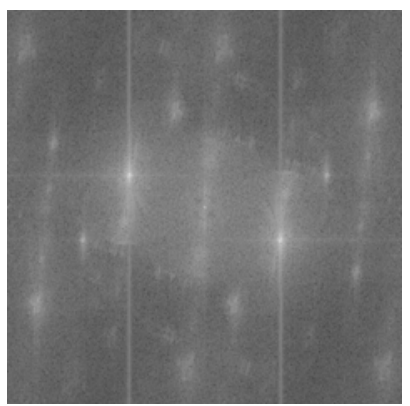

(d)

Fig. 5. Hologram. (a) the image of the onion specimen; (b), (c) are the respectively hologram with and without the onion specimen; (d) the spectrum of the hologram with the onion specimen on the logarithmic coordinates.

First, the 1D-GWT is employed for the analysis. Fig. 6(a) and (b) show the modulus and the phase of the wavelet coefficients at the $256^{\text {th }}$ row of the hologram. By detecting the ridge of the 1D-GWT and multiplying the wavelet coefficients at the ridge of the 1D-GWT by the numerical reference wave, the object wave is reconstructed. Fig. 6(c) shows the spectrum of the reconstructed wave by employing the 1D-GWT. It shows that the high frequency information of the object wave is reserved, which might be filtered out by the process of the filtering. Fig. 6(d) and (e) show the amplitude and the phase modulo $2 \pi$, coded to 256 gray levels, of the reconstructed wave. The amplitude image shown in Fig. 6(d) seems 


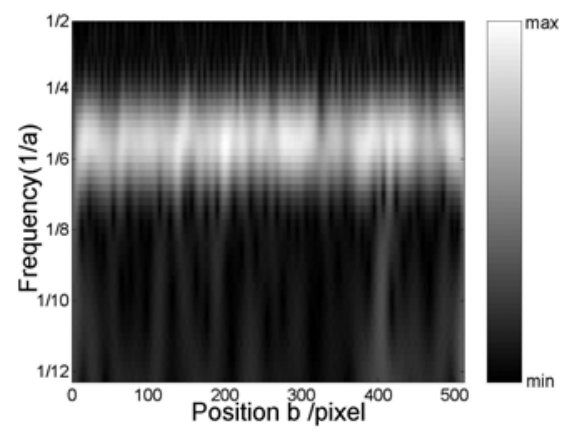

(a)

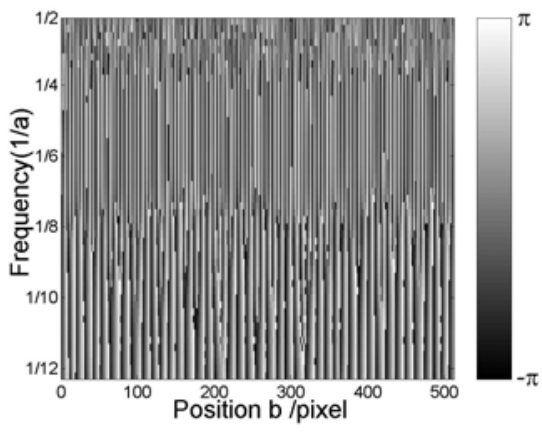

(b)

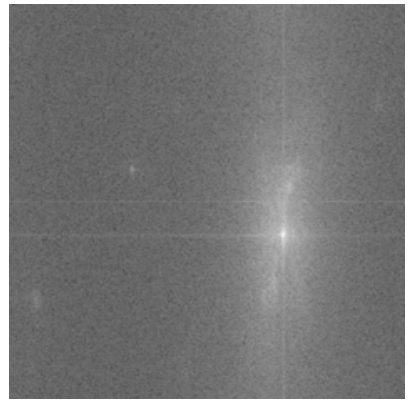

(c)

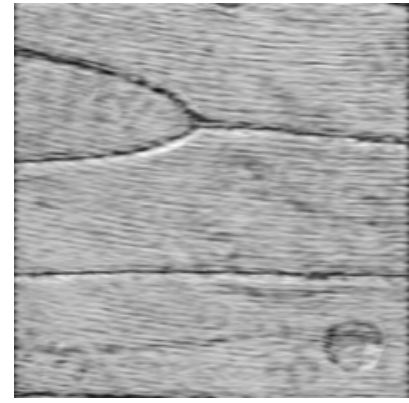

(d)

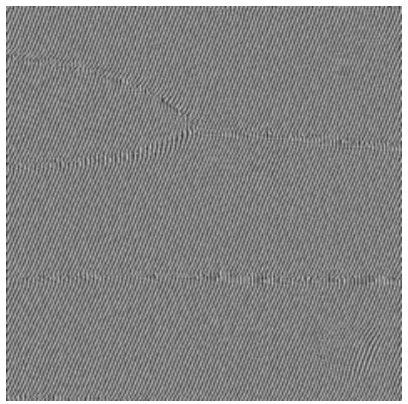

(e)

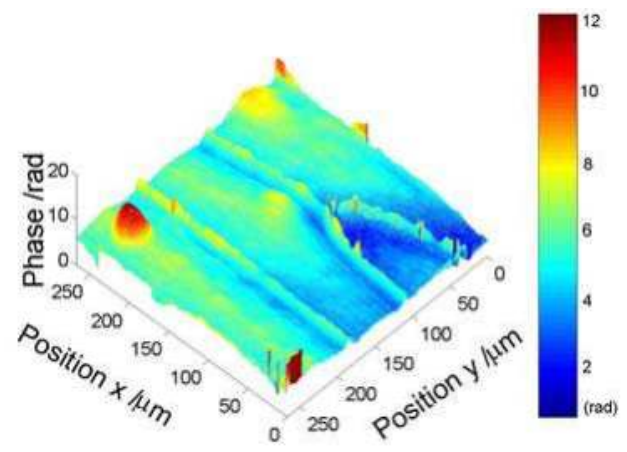

(f)

Fig. 6. Holography of an onion specimen by the analysis of the 1D-GWT. (a) the modulus and (b) the phase of the wavelet coefficients at the $256^{\text {th }}$ row of the hologram; (c) the spectrum, (d) the amplitude, (e) the wrapped phase and (f) the unwrapped phase of the reconstructed wave. 


\begin{tabular}{|c|c|c|c|c|c|c|c|c|c|c|}
\hline \multirow{2}{*}{\multicolumn{2}{|c|}{$\left|W_{I}(s, \theta, 256,256)\right|$}} & \multicolumn{9}{|c|}{$S$} \\
\hline & & 2 & 3 & 4 & 5 & 6 & 7 & 8 & 9 & 10 \\
\hline \multirow{10}{*}{$\theta$} & $0^{\circ}$ & $5.22 \mathrm{E}+00$ & $2.38 \mathrm{E}+01$ & $2.45 \mathrm{E}+01$ & $5.37 \mathrm{E}+00$ & $6.99 \mathrm{E}+00$ & $5.83 \mathrm{E}+00$ & $3.36 E+00$ & $3.33 E+00$ & $4.82 \mathrm{E}+00$ \\
\hline & $18^{\circ}$ & $3.91 E+00$ & $2.35 E+01$ & $1.13 \mathrm{E}+02$ & $1.49 E+02$ & $5.57 \mathrm{E}+01$ & $6.26 \mathrm{E}+00$ & $2.69 E+00$ & $1.36 \mathrm{E}+00$ & 7.02E-01 \\
\hline & $36^{\circ}$ & $2.51 \mathrm{E}+00$ & $1.58 \mathrm{E}+01$ & $6.70 \mathrm{E}+01$ & $7.37 \mathrm{E}+01$ & $1.97 \mathrm{E}+01$ & $6.95 E+00$ & $3.52 \mathrm{E}+00$ & 9.38E-01 & $2.12 \mathrm{E}+00$ \\
\hline & $54^{\circ}$ & $3.16 \mathrm{E}+00$ & $1.01 E+01$ & $1.19 \mathrm{E}+01$ & $9.29 \mathrm{E}+00$ & $8.86 \mathrm{E}+00$ & $5.45 \mathrm{E}+00$ & $2.36 \mathrm{E}+00$ & $3.03 \mathrm{E}+00$ & $4.36 \mathrm{E}+00$ \\
\hline & $72^{\circ}$ & $2.43 \mathrm{E}+00$ & $5.24 \mathrm{E}+00$ & $5.90 \mathrm{E}+00$ & $4.79 E+00$ & $8.69 E+00$ & $7.49 \mathrm{E}+00$ & $3.95 E+00$ & $1.11 \mathrm{E}+00$ & $4.61 \mathrm{E}+00$ \\
\hline & $90^{\circ}$ & $6.22 \mathrm{E}+00$ & $6.35 E+00$ & $1.16 \mathrm{E}+01$ & $1.34 \mathrm{E}+01$ & $1.85 \mathrm{E}+01$ & $2.74 \mathrm{E}+01$ & $2.67 E+01$ & $1.66 \mathrm{E}+01$ & $6.25 \mathrm{E}+00$ \\
\hline & $108^{\circ}$ & $1.91 \mathrm{E}+00$ & $2.01 E+00$ & $3.87 E+00$ & $3.61 E+00$ & $5.20 \mathrm{E}+00$ & $6.66 \mathrm{E}+00$ & $5.49 \mathrm{E}+00$ & $2.69 \mathrm{E}+00$ & $1.75 \mathrm{E}+00$ \\
\hline & $126^{\circ}$ & 7.92E-02 & 9.85E-02 & 1.32E-01 & $1.53 \mathrm{E}-01$ & 1.83E-01 & $1.42 \mathrm{E}-01$ & $6.80 \mathrm{E}-02$ & 6.17E-02 & 7.50E-02 \\
\hline & $144^{\circ}$ & 1.34E-03 & $1.91 \mathrm{E}-03$ & 2.00E-03 & $1.70 \mathrm{E}-03$ & 9.15E-04 & 7.14E-04 & 7.03E-04 & 4.03E-04 & 1.73E-04 \\
\hline & $162^{\circ}$ & 2.93E-04 & 3.19E-04 & 3.48E-04 & $3.66 \mathrm{E}-04$ & 3.71E-04 & 3.70E-04 & 3.71E-04 & 3.73E-04 & 3.77E-04 \\
\hline
\end{tabular}

Table 1. The modulus of the wavelet coefficients at the position $(256,256)$.

to suffer from horizontal "ripples". This "ripples" is likely to be an additional interference signal produced by the reflected wave on the slides, which appear in the image of the onion specimen shown in Fig. 5(a). Because 1D-GWT is carried out on the horizontal direction, it fails to eliminate this parasitic interference signal. It might be eliminated by employing the 2D-GWT. After removal of the $2 \Pi$ ambiguity by a phase unwrapping process, the unwrapped phase is obtained, as shown in Fig. 6(f).

In order to eliminate the "ripples" as show in Fig. 6(d), the 2D-GWT is employed for the analysis. Table 1 shows the modulus of the wavelet coefficients with nine different scale values from 2 to 10 with interval of 1 , and ten different rotation angle values from $0^{0}$ to $162^{0}$ with interval of $18^{0}$ at the position $(256,256)$. It shows that the modulus reaches the maximum when $s=5$ and $\theta=18^{0}$, i.e. the peak of the 2D-GWT of the hologram at this position is determined. Fig. $7(\mathrm{a})$, (b) and (c) show the spectrum on the logarithmic coordinates, the amplitude and the unwrapped phase of the reconstructed wave by the analysis of the 2D-GWT. From the comparison of Fig. 5(d), Fig. 6(c) and Fig. 7(a), i.e. the spectrum distribution of the hologram, the reconstructed wave by the analysis of the 1DGWT and the 2D-GWT, it can be seen that there is some unwanted spectrum by the analysis of the 1D-GWT, which results in failing to eliminate the parasitic interference signals along $x$ direction like "ripples" as show in Fig. 6(d). While there is a more accurate spectrum by the analysis of the 2D-GWT as shown in Fig. 7(a), and the result is improved by employing the 2D-GWT as show in Fig. 7(b). 


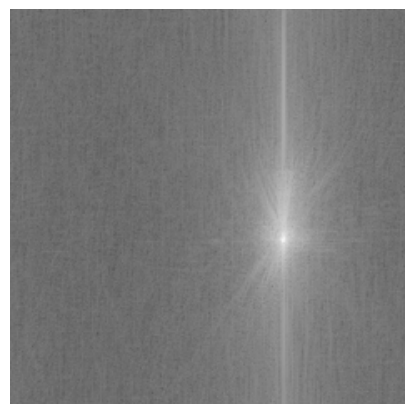

(a)

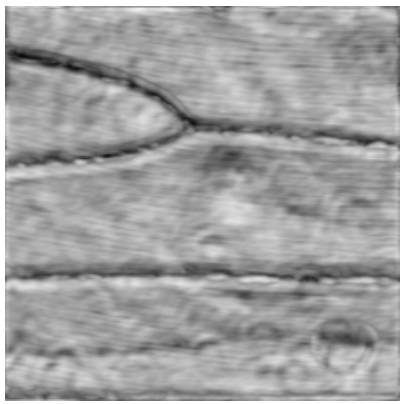

(b)

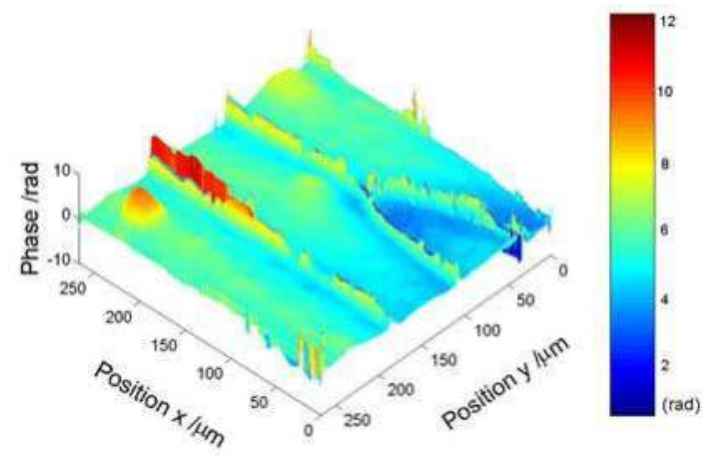

(c)

Fig. 7. Holography of an onion specimen by the analysis of the 2D-GWT. (a) the spectrum, (d) the amplitude, (b) the wrapped phase and (c) the unwrapped phase of the reconstructed wave.

\section{Conclusion}

In this chapter, the theory of the 1D-GWT and 2D-GWT techniques for the analysis of the digital holography to obtain the quantitative phase especially for the phase object are demonstrated in detail. The results of the experiment of an onion specimen show the conclusion that the numerical reconstruction of the phase object can be carried out by the analysis of the GWT. At the same time the effect of the zero-order term and the twin-image term are eliminated without the spatial filtering. One of the significant advantages of the technique here we presented is that it can be applied to the dynamic live biological specimen analysis in the digital holographic phase-contrast microscopy. 


\section{References}

Abid, A. Z.; Gdeisat, M. A.; Burton, D. R.; Lalor, M. J. \& Lilley, F. (2007). Spatial fringe pattern analysis using the two-dimensional continuous wavelet transform employing a cost function. Applied Optics, Vol. 46, No. 24, August 2007 6120-6126, ISSN 0003-6935

Anderson, W. L. \& Diao, H. (1995). Two-dimensional wavelet transform and application to holographic particle velocimetry. Applied Optics, Vol. 34, No. 2, January 1995 249255, ISSN 0003-6935

Belaïd, S.; Lebrun, D. \& Özkul, C. (1997). Application of two-dimensional wavelet transform to hologram analysis: visualization of glass fibers in a turbulent flame. Optical Engineering, Vol. 36, No. 7, July 1997 1947, ISSN 0091-3286

Carl, D.; Kemper B.; Wernicke, G. \& Bally, G. von (2004). Parameter-optimized digital holographic microscope for highresolution living-cell analysis. Applied Optics, Vol. 43, No. 36, December 2004 6536-6544, ISSN 0003-6935

Cesar, A. \& Taeeeui, K. (2003). Determination of strains from fringe patterns using spacefrequency representations. Optical Engineering, Vol. 42, No. 11, November 2003 3182-3193, ISSN 0091-3286

Colomb, T.; Cuche, E.; Charrière, F.; Kühn, J.; Aspert, N.; Montfort, F.; Marquet, P. \& Depeursinge, C. (2006). Automatic procedure for aberration compensation in digital holographic microscopy and applications to specimen shape compensation. Applied Optics, Vol. 45, No. 5, February 2006 851-863, ISSN 0003-6935

Colomb, T.; Kühn, J.; Charrière, F. \& Depeursinge, C. (2006). Total aberrations compensation in digital holographic microscopy with a reference conjugated hologram. Optics Express, Vol. 14, No. 10, May 2006 4300-4306, ISSN 1094-4087

Cuche, E.; Marquet, P. \& Depeursinge, C. (1999). Simultaneous amplitude-contrast and quantitative phase-contrast microscopy by numerical reconstruction of Fresnel offaxis holograms. Applied Optics, Vol. 38, No. 34, December 1999 6994-7001, ISSN 0003-6935

Gdeisat, M. A.; Burton, D. R. \& Lalor, M. J. (2006). Spatial carrier fringe pattern demodulation by use of a two-dimensional continuous wavelet transform. Applied Optics, Vol. 45, No. 34, December 2006 8722-8732, ISSN 0003-6935

Goodman, J. W. (1996). Introduction to Fourier Optics, Mc Graw-Hill Companies, ISBN 0-07024254-2, United States of America

Jeong, H. (2001). Analysis of plate wave propagation in anisotropic laminates using a wavelet transform. NDT \& E International, Vol. 34, No. 3, April 2001 185-190, ISSN 0963-8695

Kadooka, K.; Kunoo, K.; Uda, N.; Ono, K. \& Nagayasu, T. (2003). Strain analysis for moiré interferometry using the two-dimensional continuous wavelet transform. Experimental Mechanics, Vol. 43, No. 1, March 2003 45-51, ISSN 0014-4851

Kemper, B. \& Bally, G. von (2008). Digital holographic microscopy for live cell applications and technical inspection. Applied Optics, Vol. 47, No. 4, February 2008 A52-A61, ISSN 0003-6935

Kühn, J.; Colomb, T.; Montfort, F.; Charrière, F.; Emery, Y.; Cuche, E.; Marquet, P. \& Depeursinge, C. (2007). Real-time dual-wavelength digital holographic microscopy 
with a single hologram acquisition. Optics Express, Vol. 15, No. 12, June 2007 72317242, ISSN 1094-4087

Langehanenberg, P.; Kemper, B.; Dirksen, D. \& Bally, G. von (2008). Autofocusing in digital holographic phase contrast microscopy on pure phase objects for live cell imaging. Applied Optics, Vol. 47, No. 19, July 2008 D176-D182, ISSN 0003-6935

Lebrun, D.; Belaïd, S. \& Özkul, C. (1999). Hologram reconstruction by use of optical wavelet transform. Applied Optics, Vol. 38, No. 17, June 1999 3730-3734, ISSN 0003-6935

Li, Zh.; Gu, B. \& Yang, G. (1999). Slowly varying amplitude approximation appraised by transfer-matrix approach. Physical Review B, Vol. 60, No. 15, October 199910644 10647, ISSN 0163-1829

Liebling, M.; Blu, T. \& Unser, M. (2003). Fresnelets: new multiresolution wavelet bases for digital holography. IEEE Transactions on Image Processing, Vol. 12, No. 1, January 2003 29-43, ISSN 1057-7149

Mann, C. J.; Yu, L.; Lo, C. \& Kim, M. K. (2005). High-resolution quantitative phase-contrast microscopy by digital holography. Optics Express, Vol. 13, No. 22, October 2005 8693-8698, ISSN 1094-4087

Marquet, P.; Rappaz, B.; Magistretti, P. J.; Cuche, E.; Emery, Y.; Colomb, T.; \& Depeursinge, C. (2005). Digital holographic microscopy: a noninvasive contrast imaging technique allowing quantitative visualization of living cells with subwavelength axial accuracy. Optics Letters, Vol. 30, No. 5, March 2005 468-470, ISSN 0146-9592

Montfort, F.; Charrière, F. \& Colomb, T. (2006). Purely numerical compensation for microscope objective phase curvature in digital holographic microscopy: influence of digital phase mask position. Journal of the Optical Society of America A, Vol. 23, No. 11, November 2006 2944-2953, ISSN 1520-8532

Shimobaba, T.; Sato, Y.; Miura, J.; Takenouchi, M. \& Ito, T. (2008). Real-time digital holographic microscopy using the graphic processing unit. Optics Express, Vol. 16, No. 16, August 2008 11776-11781, ISSN 1094-4087

Wang, Z. \& Ma, H. (2006). Advanced continuous wavelet transform algorithm for digital interferogram and processing. Optical Engineering, Vol. 45, No. 4, April 2006 1-5, ISSN 0091-3286

Weng, J.; Zhong, J. \& Hu, C. (2008). Digital reconstruction based on angular spectrum diffraction with the ridge of wavelet transform in holographic phase-contrast microscopy. Optics Express, Vol. 16, No. 26, December 2008 21971-21981, ISSN 10944087

Weng, J.; Zhong, J. \& Hu, C. (2009). Phase reconstruction of digital holography with the peak of the two-dimensional Gabor wavelet transform. Applied Optics, Vol. 48, No. 18, June 2009 3308-3316, ISSN 0003-6935

Weng, J.; Zhong, J. \& Hu, C. (2010). Automatic spatial filtering to obtain the virtual image term in digital holographic microscopy. Applied Optics, Vol. 49, No. 2, January 2010 189-195, ISSN 0003-6935

Yu, L. \& Kim, M. K. (2005). Wavelength-scanning digital interference holography for tomographic three-dimensional imaging by use of the angular spectrum method. Optics Letters, Vol. 30, No. 16, August 2005 2092-2094, ISSN 0146-9592 
Zhong, J. \& Weng, J. (2004). Spatial carrier-fringe pattern analysis by means of wavelet transform: wavelet transform profilomatry. Applied Optics, Vol. 43, No. 26, September 2004 4993-4998, ISSN 0003-6935

Zhong, J. \& Weng, J. (2005). Phase retrieval of optical fringe pattern form the ridge of a wavelet transform. Optics Letters, Vol. 30, No. 19, October 2005 2560-2562, ISSN 0146-9592 


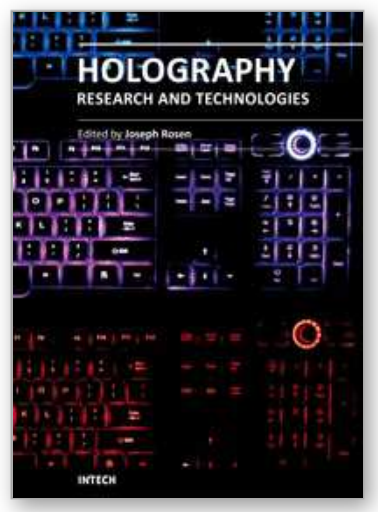

\author{
Holography, Research and Technologies \\ Edited by Prof. Joseph Rosen
}

ISBN 978-953-307-227-2

Hard cover, 454 pages

Publisher InTech

Published online 28, February, 2011

Published in print edition February, 2011

Holography has recently become a field of much interest because of the many new applications implemented by various holographic techniques. This book is a collection of 22 excellent chapters written by various experts, and it covers various aspects of holography. The chapters of the book are organized in six sections, starting with theory, continuing with materials, techniques, applications as well as digital algorithms, and finally ending with non-optical holograms. The book contains recent outputs from researches belonging to different research groups worldwide, providing a rich diversity of approaches to the topic of holography.

\title{
How to reference
}

In order to correctly reference this scholarly work, feel free to copy and paste the following:

Jingang Zhong and Jiawen Weng (2011). Reconstruction of Digital Hologram by use of the Wavelet Transform, Holography, Research and Technologies, Prof. Joseph Rosen (Ed.), ISBN: 978-953-307-227-2, InTech, Available from: http://www.intechopen.com/books/holography-research-andtechnologies/reconstruction-of-digital-hologram-by-use-of-the-wavelet-transform

\section{INTECH}

open science | open minds

\section{InTech Europe}

University Campus STeP Ri

Slavka Krautzeka 83/A

51000 Rijeka, Croatia

Phone: +385 (51) 770447

Fax: +385 (51) 686166

www.intechopen.com

\section{InTech China}

Unit 405, Office Block, Hotel Equatorial Shanghai

No.65, Yan An Road (West), Shanghai, 200040, China

中国上海市延安西路 65 号上海国际贵都大饭店办公楼 405 单元

Phone: +86-21-62489820

Fax: +86-21-62489821 
(C) 2011 The Author(s). Licensee IntechOpen. This chapter is distributed under the terms of the Creative Commons Attribution-NonCommercialShareAlike-3.0 License, which permits use, distribution and reproduction for non-commercial purposes, provided the original is properly cited and derivative works building on this content are distributed under the same license. 